\title{
AVALIAÇÃO DE ESTRATÉGIAS DE SINTONIAS DO DMC
}

\author{
H. C. OYAMA ${ }^{1}$, L. C. OLIVEIRA-LOPES ${ }^{1}$ \\ ${ }^{1}$ Universidade Federal de Uberlândia, Faculdade de Engenharia Química \\ E-mail para contato: henrique.oyama@ufu.br
}

\begin{abstract}
RESUMO - A sintonia de algoritmos de controle multivariáveis, em especial para o MPC, caracterizado pela complexidade nas combinações dos efeitos de seus parâmetros, ainda é palco de estudos para contribuir no desempenho e robustez dessa categoria de controladores. Nesse viés, o objetivo deste trabalho foi investigar diretrizes de sintonias do controlador preditivo DMC e selecionar a melhor metodologia através de índices de desempenho (ITAE e RMS). Os métodos mais convenientes atestados aos problemas servo e regulador foram o M3 e o M7 (uma extensão de M3) aplicados ao sistema SISO e MIMO, respectivamente.
\end{abstract}

\section{INTRODUÇÃO}

Nas décadas recentes, o Controle Preditivo Baseado em Modelo (MPC) se consagrou como a forma mais insigne de controle avançado no setor industrial, sendo que o controlador do tipo Controle por Matriz Dinâmica (DMC) em horizonte finito é considerado um dos algoritmos mais populares utilizados na indústria química (Shridhar e Cooper, 1998).

Apesar do conjunto de vantagens do MPC descritas por Campos, Gomes e Perez (2013), a dependência do modelo do processo e, em especial, a elevada combinação de parâmetros de sintonia constituem os grandes reptos da área. Assim, como é comum na literatura e em razão da dificuldade de definição de índices de desempenho em operações com restrições ativas, este trabalho tem como objetivo investigar e avaliar métodos de sintonia de controladores preditivos (DMC) sem restrições para o Horizonte de Predição e Horizonte de Controle, e cotejar os resultados das diversas diretrizes através de índices de desempenho (ITAE e RMS).

\section{A LEI DE CONTROLE DO DMC}

O problema de otimização em horizonte finito do MPC é resolvido através da minimização de uma função custo (J), dada pela Equação 1, aqui avaliada sem restrições, modificada para penalizar os erros das variáveis de maior importância ao processo; e a Equação 2 que representa a lei de controle obtida para o caso de um problema multivariável, com $n y$ variáveis controladas e $n u$ variáveis manipuladas (Campos, Gomes e Perez, 2013).

$$
\begin{aligned}
& \operatorname{mí}_{\Delta U} J=E^{T} W^{T} W E+\Delta U^{T} R \Delta U \\
& \Delta U=\left(S^{T} W^{T} W S+R\right)^{-1} S^{T} W^{T} W(w-f)
\end{aligned}
$$


Os principais parâmetros de sintonia do controlador preditivo do tipo DMC são: Horizonte de Predição (Hp); Horizonte de Controle (Hc); pesos nas variáveis manipuladas (R - Matriz de Supressão de Movimento) e pesos nas variáveis controladas (W ou Equal Concern, $\mathrm{EC}=1 / \mathrm{W})$.

\subsection{Sintonia para o DMC}

Para este estudo preliminar, os pesos nas variáveis controladas foram fixados no valor unitário e a Matriz de Supressão de Movimento foi definida pelo método de Shridhar e Cooper (1998), conforme a Equação 3.

$$
R_{s}=\frac{H c}{500} \sum_{r=1}^{n y}\left[W_{r} K_{r s}^{2}\left\{H p-k_{r s}-\frac{3}{2} \frac{\tau_{r s}}{T s}+2-\frac{(H c-1)}{2}\right\}\right], s=1,2, \ldots, n u ; r=1,2, \ldots, n y
$$

Os métodos de sintonia para o DMC foram avaliados em dois cenários, monovariável (SISO) e multivariável (MIMO), e com tempo de amostragem Ts, de acordo com as diretrizes da Tabela 1.

Tabela 1 - Métodos de Sintonia do Horizonte de Predição e Horizonte de Controle.

\begin{tabular}{|c|c|c|c|}
\hline Método & Valor ou Banda & Sistema & Referência \\
\hline M1 & $\begin{array}{c}H p=\left(t_{60}+t_{95}\right) / T s-1 \\
H c=t_{60} / T s\end{array}$ & MIMO & Georgiou, Georgakis e Luybes, 1988 \\
\hline M2 & $H p=H c+N$ & MIMO & $\begin{array}{c}\text { Cutler, 1983, apud Maurath, } \\
\text { Mellichamp e Seborg, 1988, p.958 }\end{array}$ \\
\hline M3 & $\begin{array}{c}H p=(5 \tau c+t d) / T s \\
H c=\tau c / T s\end{array}$ & SISO & Hinde Jr e Cooper, 1994 \\
\hline M4 & $\begin{array}{c}H p=\max \left(5 \tau_{r s} / T s+k_{r s}\right) \\
H c=\max \left(\tau_{r s} / T s+k_{r s}\right) \\
k_{r s}=t_{d, r s} / T s+1\end{array}$ & MIMO & Shridhar e Cooper, 1998 \\
\hline M5 & $H p=\left[\left(t_{80}+t_{90}\right) / 2\right] / T s$ & MIMO & Maurath et al., 1988 \\
\hline M6 & $H p>H c+t d / T s$ & SISO & Maurath, Mellichamp e Seborg, 1988 \\
\hline M7 & $\begin{array}{c}H p=\max \left(5 \max \left(\tau_{r s}\right)+\min \left(t_{d, r s}\right) / T s\right) \\
H c=\max \left(\tau_{r s}\right) / T s\end{array}$ & MIMO & Adaptado de M3 \\
\hline M8 & $H p>H c+\min \left(t_{d, r s}\right) / T s$ & MIMO & Adaptado de M6 \\
\hline
\end{tabular}

\section{2. Índices de Desempenho de Sistemas Controlados}

Os índices de desempenho adotados nesse trabalho foram a integral ponderada pelo tempo para o valor absoluto do erro (ITAE) e a raiz do erro quadrático médio (RMS), indicadas na forma discretizada pelas Equações 4 e 5, respectivamente.

$$
\begin{aligned}
\operatorname{ITAE} & =\sum_{k=0}^{T} k|e(k)| \\
\mathrm{RMS} & =\sqrt{1 /(T+1) \sum_{k=0}^{T} e(k)^{2}}
\end{aligned}
$$

Em que $T$ representa o número de Ts correspondente ao tempo total avaliado. 


\subsection{Avaliação dos Sistemas Controlados}

O sistema de controle SISO investigado corresponde ao controle de um processo estável de um trocador de calor, conforme apresentado por Camacho e Bordons (1999). O tempo de amostragem foi de 0,6 segundos e a relação entre a temperatura de saída (Y) e a vazão de entrada no trocador de calor (u) é dada pela função de transferência da Equação 6. Avaliaramse as diversas sintonias com base nos índices de desempenho ITAE e RMS aplicados a um problema servo, em que se realizou uma perturbação degrau unitário no setpoint no instante 5, e a um problema regulador, no qual se introduziu uma perturbação no estado da planta no instante 10 .

$$
P(s)=\frac{0,12 e^{-3 s}}{1+6 s}
$$

Para a avaliação do problema de controle de um sistema estável MIMO foi investigado o processo de uma coluna de destilação. O fracionador de óleo pesado descrito por Camacho e Bordons (1999) apresenta três variáveis controladas - composição do produto no topo (Y1), composição do produto no prato intermediário (Y2) e temperatura no fundo da coluna (Y3) e três variáveis manipuladas - taxa de retirada no topo (u1), taxa de retirada no prato intermediário (u2) e refluxo no refervedor (u3). O tempo de amostragem foi de 4 minutos e a dinâmica do processo pode ser caracterizada pelo sistema a seguir (com Número de Condicionamento de 24,33):

$$
\left[\begin{array}{l}
Y 1(s) \\
Y 2(s) \\
Y 3(s)
\end{array}\right]=\left[\begin{array}{ccc}
\frac{4,05 e^{-27 s}}{1+50 s} & \frac{1,77 e^{-28 s}}{1+60 s} & \frac{5,88 e^{-27 s}}{1+50 s} \\
\frac{5,39 e^{-18 s}}{1+50 s} & \frac{5,72 e^{-14 s}}{1+60 s} & \frac{6,9 e^{-15 s}}{1+40 s} \\
\frac{4,38 e^{-20 s}}{1+33 s} & \frac{4,42 e^{-22 s}}{1+44 s} & \frac{7,2}{1+19 s}
\end{array}\right]\left[\begin{array}{l}
u 1(s) \\
u 2(s) \\
u 3(s)
\end{array}\right]
$$

Como critério de avaliação, adotou-se o mesmo do caso SISO, sendo que os índices de desempenho foram calculados pela combinação dos respectivos ITAE e RMS para as variáveis controladas e manipuladas, segundo as Equações 8 e 9, em que $\beta_{i}$ e $\gamma_{i}$ correspondem ao inverso da unidade de $I T A E_{i}$ (Equação 8) e $R M S_{i}$ (Equação 9), respectivamente. Além disso, foi também realizada uma extensão dos métodos M3 e M6 para este novo cenário (M7 e M8). O problema servo foi simulado com as perturbações nos setpoints no instante 10, levando-os aos valores de referência 0,5, 0,3 e 0,1 para Y1, Y2 e Y3, nesta sequência, e o problema regulador, através de simulação análoga ao sistema SISO.

$$
\begin{aligned}
& I T A E g=\sum_{i=1}^{3} \beta_{i} I T A E_{i} \\
& R M S g=\sum_{i=1}^{3} \gamma_{i} R M S_{i}
\end{aligned}
$$

\section{RESULTADOS E DISCUSSÃO}

Fixado $R_{1}=1$ como valor que compõe a Supressão de Movimento do problema de controle monovariável, calculada a partir da Equação 5, foi simulada a implementação do sistema de controle do trocador de calor, conforme os resultados resumidos na Tabela 2 para 0 problema servo (colunas "Sv") e regulador (colunas "Rg"). Ademais, utilizou-se um 
algoritmo de evolução diferencial de maneira a obter o melhor par Hp/Hc que minimiza ITAE da variável controlada do problema servo (linha "MO"). O tempo de simulação foi de 150 instantes de amostragem.

Tabela 2 - Resultados do problema servo e regulador para o sistema SISO.

\begin{tabular}{|c|c|c|c|c|c|c|c|c|c|}
\hline Método & Par & \multicolumn{2}{|c|}{ ITAE } & \multicolumn{2}{c|}{ ITAE } & \multicolumn{2}{c|}{ RMS } & \multicolumn{2}{c|}{ RMS } \\
\hline Hp/Hc & Hp/Hc & Sv-Y & Rg-Y & Sv-u & Rg-u & Sv-Y & Rg-Y & Sv-u & Rg-u \\
\hline M1/M1 & $57 / 23$ & 384,10 & 44,33 & 1076,70 & 186,03 & 0,33 & 0,08 & 1,82 & 0,18 \\
\hline M2/M2 & $51 / 15$ & 378,71 & 44,24 & 953,38 & 178,83 & 0,33 & 0,08 & 1,83 & 0,19 \\
\hline M3/M3 & $\mathbf{5 5 / 1 0}$ & $\mathbf{3 5 7 , 7 2}$ & $\mathbf{4 2 , 7 6}$ & $\mathbf{9 1 8 , 6 6}$ & $\mathbf{1 7 3 , 7 6}$ & $\mathbf{0 , 3 2}$ & $\mathbf{0 , 0 8}$ & $\mathbf{1 , 7 7}$ & $\mathbf{0 , 1 9}$ \\
\hline M4/M4 & $52 / 12$ & 369,03 & 43,38 & 979,05 & 178,21 & 0,33 & 0,08 & 1,79 & 0,19 \\
\hline M5/M1 & $32 / 23$ & 386,55 & 44,65 & 1113,35 & 189,61 & 0,33 & 0,08 & 1,83 & 0,18 \\
\hline M5/M2 & $32 / 15$ & 384,05 & 44,52 & 1101,17 & 188,81 & 0,33 & 0,08 & 1,82 & 0,19 \\
\hline M5/M3 & $32 / 10$ & 372,21 & 44,01 & 1053,21 & 186,17 & 0,33 & 0,08 & 1,80 & 0,19 \\
\hline M5/M4 & $32 / 12$ & 377,77 & 44,30 & 1074,06 & 187,53 & 0,40 & 0,08 & 2,22 & 0,19 \\
\hline M6/M1 & $28 / 23$ & 393,89 & 45,08 & 1157,43 & 192,93 & 0,33 & 0,08 & 1,84 & 0,18 \\
\hline M6/M2 & $20 / 15$ & 466,81 & 47,70 & 1437,55 & 205,60 & 0,34 & 0,08 & 1,96 & 0,16 \\
\hline M6/M3 & $15 / 10$ & 673,39 & 51,52 & 2099,76 & 218,67 & 0,38 & 0,08 & 2,27 & 0,13 \\
\hline M6/M4 & $17 / 12$ & $\mathbf{5 5 4 , 8 2}$ & 49,59 & 1707,89 & 211,22 & 0,36 & 0,08 & 2,10 & 0,14 \\
\hline MO & $\mathbf{4 3 / 5}$ & $\mathbf{3 0 8 , 4 4}$ & $\mathbf{4 8 , 8 4}$ & $\mathbf{7 7 5 , 8 8}$ & $\mathbf{2 6 0 , 3 5}$ & $\mathbf{0 , 3 1}$ & $\mathbf{0 , 0 2}$ & $\mathbf{1 , 6 8}$ & $\mathbf{0 , 1 5}$ \\
\hline
\end{tabular}

De acordo com a Tabela 2, a melhor combinação dos parâmetros de sintonia (Hp e Hc) para os problemas servo e regulador foi ensejada pelo método M3 de Hinde Jr e Cooper (1994), em que os índices de desempenho (ITAE e RMS) avaliados para as controladas e manipuladas alcançaram os menores valores entre os métodos investigados, passando 15,98\% e 18,40\% em relação ao ITAE de "Y" e "u", respectivamente, da melhor combinação de parâmetros do problema servo (par 43/5). Porém, quando aplicado ao problema regulador, o par de MO não suscitou uma melhor sintonia quando comparado ao M3. O resultado gráfico do controle servo com a seleção desses parâmetros da estratégia M3 é mostrado na Figura 1.

Figura 1 - Resultado da simulação com Hp e Hc obtidos por M3 do cenário SISO para as variáveis controladas (a) e manipuladas (b).

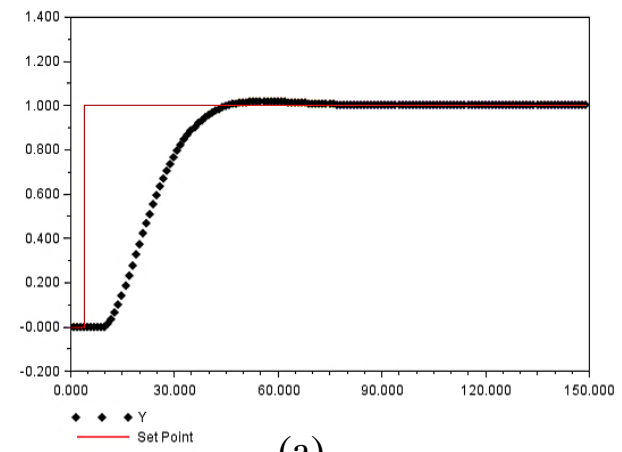

(a)

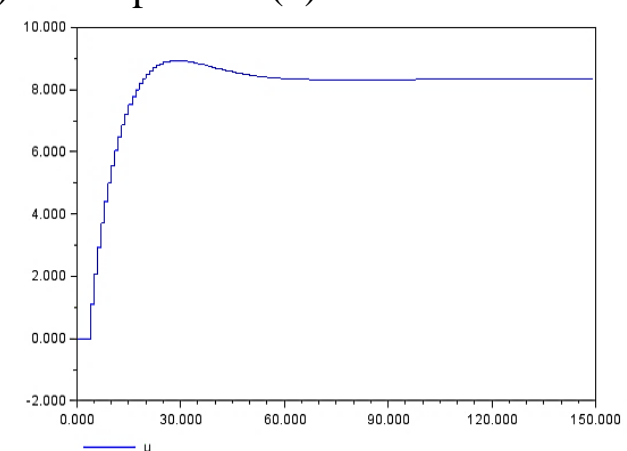

(b)

De forma análoga ao sistema multivariável, mediante a Equação 5, foram fixados $R_{1}=96, R_{2}=82$ e $R_{3}=269$ como os elementos que compõem a diagonal da Matriz dos Pesos nas Manipuladas e foi simulado a implementação do controle da coluna de destilação com tempo de simulação de 200 instantes de amostragem. A síntese dos resultados é apresentada na Tabela 3 como solução dos dois problemas típicos de controle avaliados. 
O método M7 (extensão proposta de M3) foi proficiente para o sistema de controle MIMO aplicado aos problemas servo e regulador, proporcionando as melhores medidas quantitativas de desempenho, próximas da combinação encontrada pelo algoritmo de otimização engendrado para o problema servo. O resultado na forma gráfica do controle servo com o par de parâmetros obtidos por M7 é apresentado na Figura 2.

Tabela 3 - Resultados do problema servo e regulador para o sistema MIMO.

\begin{tabular}{|c|c|c|c|c|c|c|c|c|c|}
\hline Método & Par & \multicolumn{2}{|c|}{ ITAEg } & \multicolumn{2}{c|}{ ITAEg } & \multicolumn{2}{c|}{ RMSg } & \multicolumn{2}{c|}{ RMSg } \\
\hline Hp/Hc & Hp/Hc & Sv-Y & Rg-Y & Sv-u & Rg-u & Sv-Y & Rg-Y & Sv-u & Rg-u \\
\hline M1/M1 & $72 / 29$ & 662,13 & 439,29 & 496,13 & 188,12 & 0,24 & 0,23 & 0,19 & 0,05 \\
\hline M2/M2 & $65 / 20$ & 648,27 & 441,50 & 497,67 & 190,53 & 0,24 & 0,23 & 0,19 & 0,05 \\
\hline M7/M7 & $\mathbf{8 2 / 1 5}$ & $\mathbf{5 9 7 , 6 2}$ & $\mathbf{4 3 4 , 1 6}$ & $\mathbf{3 9 0 , 2 2}$ & $\mathbf{1 7 5 , 9 6}$ & $\mathbf{0 , 2 4}$ & $\mathbf{0 , 2 3}$ & $\mathbf{0 , 1 7}$ & $\mathbf{0 , 0 5}$ \\
\hline M4/M4 & $81 / 21$ & 628,13 & 436,92 & 430,89 & 179,71 & 0,24 & 0,23 & 0,17 & 0,05 \\
\hline M5/M1 & $40 / 29$ & 911,72 & 443,48 & 818,87 & 197,99 & 0,24 & 0,23 & 0,21 & 0,05 \\
\hline M5/M2 & $40 / 20$ & 904,02 & 442,69 & 809,53 & 197,45 & 0,24 & 0,23 & 0,21 & 0,05 \\
\hline M5/M7 & $40 / 15$ & 909,84 & 441,37 & 815,85 & 196,23 & 0,24 & 0,23 & 0,21 & 0,05 \\
\hline M5/M4 & $40 / 21$ & 904,02 & 442,86 & 809,53 & 197,63 & 0,24 & 0,23 & 0,21 & 0,05 \\
\hline M8/M1 & $29 / 29$ & 1336,67 & 444,09 & 1399,57 & 181,56 & 0,25 & 0,23 & 0,24 & 0,05 \\
\hline M8/M2 & $20 / 20$ & 1678,28 & 440,55 & 1786,55 & 162,76 & 0,25 & 0,23 & 0,26 & 0,04 \\
\hline M8/M7 & $15 / 15$ & 1459,91 & 438,70 & 1639,08 & 175,66 & 0,26 & 0,22 & 0,27 & 0,04 \\
\hline M8/M4 & $21 / 21$ & 1667,96 & 441,19 & 1772,32 & 164,17 & 0,25 & 0,23 & 0,26 & 0,04 \\
\hline MO & $\mathbf{8 2 / 1 1}$ & $\mathbf{5 8 1 , 1 5}$ & $\mathbf{2 1 0 , 1 5}$ & $\mathbf{3 6 7 , 4 5}$ & $\mathbf{9 2 , 0 2}$ & $\mathbf{0 , 2 4}$ & $\mathbf{0 , 1 1}$ & $\mathbf{0 , 1 7}$ & $\mathbf{0 , 0 3}$ \\
\hline
\end{tabular}

Figura 2 - Resultado da simulação com Hp e Hc obtidos por M7 do cenário MIMO para as variáveis controladas (a) e manipuladas (b).
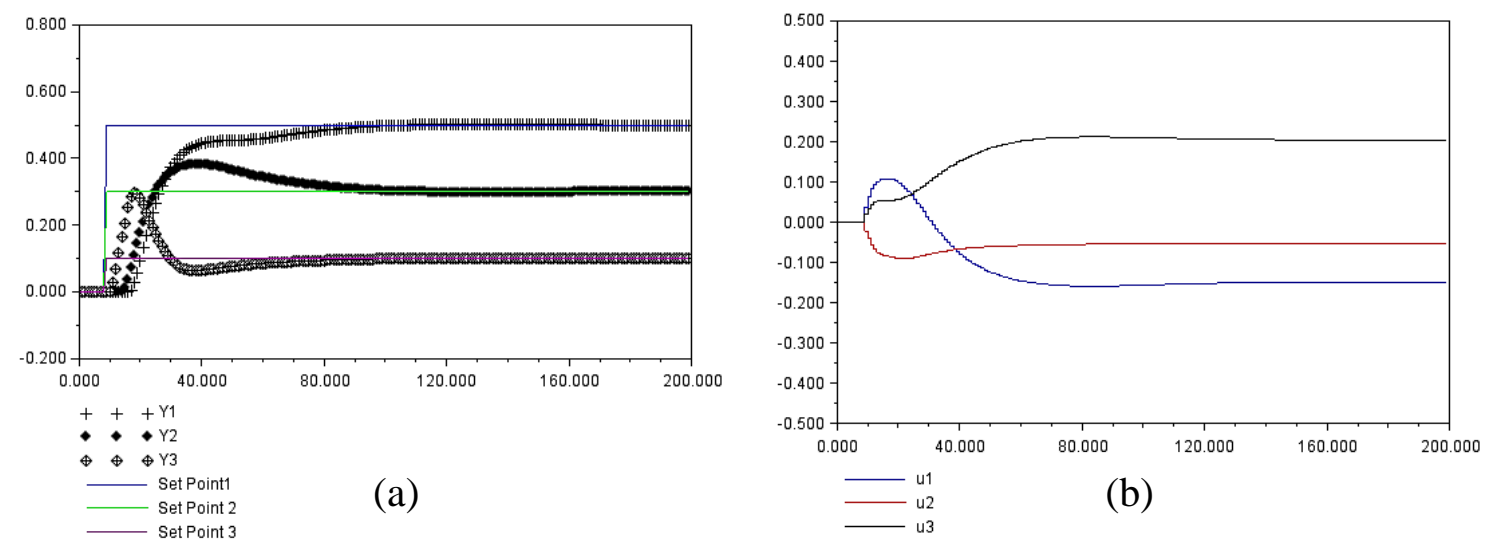

Conforme esperado, verificou-se, segundo a Tabela 2, que as extraviadas sintonias do controlador foram aquelas obtidas da combinação com M6, resvalando os maiores dispêndios em termos de desempenho para o problema regulador e, em especial, para o problema servo (os erros de "u" e "Y" que ocorreram mais tarde na resposta foram os mais acentuados). Por outro lado, entre as diretrizes estudadas ao cenário MIMO, a combinação de M8 (adaptado de M6, ambos aplicados no limite da igualdade) com M2, M4 ou M7 se mostrou vantajoso apenas para o problema regulador. Em relação às medidas de desempenho, notou-se que nos dois cenários o RMS não conduziu a uma definitiva seletividade (valores muito próximos) para os métodos avaliados, sendo o ITAE o critério diferenciador nesse estudo. 


\section{CONCLUSÃO}

Entre as diretrizes de sintonia contempladas neste trabalho, o M3 de Hinde Jr e Cooper (1994) para um sistema SISO e a proposta de estender este método para um sistema MIMO (M7) amoldaram os melhores índices de desempenho - ITAE e RMS (critério com baixa seletividade) - desvelados a um problema servo e regulador.

Conforme esperado, as combinações das estratégias com M6 e com M8, por serem definidas mediante bandas de valores não garantiram uma sintonia eficaz ao cenário proposto, o que representa, amiúde, resultados indesejáveis com índices de desempenho elevados. Contudo, o método M8 coadunado com M2, M4 ou M7 se apresentou profícuo somente quando aplicado a um problema regulador.

Para avaliar outras combinações possíveis de ajustes que podem matizar melhores efeitos no desempenho e robustez do controlador, sugere-se, como trabalho vindouro, o estudo de métodos de sintonia para o peso nas variáveis controladas e manipuladas (tanto para modelos de convolução quanto para modelos em espaço de estados), em consonância com as sintonias investigadas neste trabalho, seja feito inclusive para sistemas não quadrados.

\section{AGRADECIMENTOS}

Os autores agradecem à UFU e ao CNPQ pelo apoio concedido.

\section{BIBLIOGRAFIA}

CAMACHO, E. F.; BORDONS, C. Model Predictive Control. 2. ed. Sevilha: Springer, 1999.

CAMPOS, M. C. M. M. D.; GOMES, M. V. D. C.; PEREZ, J. M. G. T. Controle Avançado e Otimização na Indústria do Petróleo. Rio de Janeiro: Interciência, 2013.

GEORGIOU, A.; GEORGAKIS, C.; LUYBEN, W. L. Nonlinear Dynamic Matrix Control for High-Purity Distillation Columns. AIChE Journal, v. 34, n. 8, p. 1287-1298, 1988.

HINDE JR, R. F.; COOPER, D. J. A pattern-based approach to excitation diagnostics for adaptive process control. Chemical Engineering Science, v. 49, n. 9, p. 1403-1415, 1994.

MAURATH, P. R. et al. Predictive controller design by principal components analysis. Industrial and Engineering Chemistry Research, v. 27, n. 7, p. 1204-1212, 1988.

MAURATH, P. R.; MELLICHAMP, D. A.; SEBORG, D. E. Predictive controller design for single-input/single-output (SISO) systems. Industrial and Engineering Chemistry Research, v. 27, n. 6, p. 956-963, 1988.

SHRIDHAR, R.; COOPER, D. J. A tuning strategy for unconstrained multivariable model predictive control. Industrial and Engineering Chemistry Research, v. 37, n. 10, p. 4003-4016, 1998. 